\title{
Multiple Cranial Neuropathies
}

\author{
Craig G. Carroll, D.O., ${ }^{1}$ and William W. Campbell, M.D., M.S.H.A. ${ }^{2}$
}

\begin{abstract}
Patients presenting with multiple cranial neuropathies are not uncommon in neurologic clinical practice. The evaluation of these patients can often be overwhelming due to the vast and complicated etiologies as well as the potential for devastating neurologic outcomes. Dysfunction of the cranial nerves can occur anywhere in their course from intrinsic brainstem dysfunction to their peripheral courses. The focus of this review will be on the extramedullary causes of multiple cranial neuropathies as discussion of the brainstem syndromes is more relevant when considering intrinsic disorders of the brainstem. The goals are to provide the reader with an overview of those extramedullary conditions that have a predilection for causing multiple cranial nerve palsies. In turn, this will serve to provide a practical and systematic approach to allow for a more targeted diagnostic evaluation of this, often cumbersome, presentation.
\end{abstract}

KEYWORDS: Multiple cranial neuropathy, cranial neuropathies, cranial nerve palsies, cranial nerve syndromes, cranial polyneuropathies

Evaluating the patient with multiple cranial neuropathies presents a unique challenge for the diagnostician. The differential diagnosis is broad and includes many life-threatening processes. Just as with any other neurologic presentation, the first step in the evaluation requires correct localization. Processes affecting multiple cranial nerves may involve intramedullary structures of the brainstem as well as their extramedullary course. The focus of this review will be on the extramedullary disorders affecting multiple cranial nerves.

\section{BRAINSTEM SYNDROMES}

Prior to discussion of the extramedullary processes causing dysfunction of multiple cranial nerves, a few points must be made about the brainstem syndromes. Many of the early neurologic pioneer clinicians described the findings due to a focal process affecting the brain- stem, and thus brainstem syndromes are often organized into eponymic syndromes. However, these classically described eponymic brainstem syndromes were reported in an era where disorders such as tuberculomas, syphilitic gummas, and tumors were seen more often than today. ${ }^{1}$ Many of these classically described brainstem syndromes were not due to ischemia. In the current era, the vast majority of brainstem syndromes are a result of vascular insults, mainly brainstem infarctions and hemorrhages, often involving the lateral pons and medulla. ${ }^{2}$ Considering the dominance of vascular etiologies of the brainstem syndromes, a more practical classification of these syndromes is by the anatomical area or the major blood vessel involved (Table 1). Examples of nonvascular disorders that commonly affect the brainstem include demyelinating disease (multiple sclerosis [MS], acute disseminated encephalomyelitis [ADEM]), intramedullary neoplasms (such as brainstem gliomas/ependymomas), brainstem encephalitis (Bickerstaff's encephalitis),
${ }^{1}$ Department of Neurology, Naval Medical Center, Portsmouth, Virginia; ${ }^{2}$ Department of Neurology, Uniformed Services University of Health Sciences, Bethesda, Maryland.

Address for correspondence and reprint requests: Craig G. Carroll, D.O., Head, Department of Neurology, Naval Medical Center Portsmouth, 620 John Paul Jones Circle, Portsmouth, VA 23708 (e-mail: craig.carroll@med.navy.mil).
Disorders of the Cranial Nerves; Guest Editor, William W. Campbell, M.D., M.S.H.A.

Semin Neurol 2009;29:53-65. Copyright (C) 2009 by Thieme Medical Publishers, Inc., 333 Seventh Avenue, New York, NY 10001, USA. Tel: +1(212) 584-4662.

DOI 10.1055/s-0028-1124023. ISSN 0271-8235. 
Table 1 Summary of the Brainstem Syndrome Organized by Anatomic Region and Blood Vessel Involved

\begin{tabular}{|c|c|c|c|}
\hline Syndrome & Structures Involved & Clinical Findings & Comments \\
\hline Midbrain syndromes & $\begin{array}{l}\text { Corticospinal tract; corticobulbar } \\
\text { tract; cranial nerve III fibers; } \\
\text { red nucleus; superior cerebellar } \\
\text { peduncle }\end{array}$ & $\begin{array}{l}\text { Contralateral weakness of arm, } \\
\text { leg and face; ipsilateral cranial } \\
\text { nerve III; contralateral tremor; } \\
\text { contralateral ataxia }\end{array}$ & $\begin{array}{l}\text { Due to occlusion of the interpeduncular } \\
\text { penetrating branches of the basilar } \\
\text { or posterior cerebral artery or the } \\
\text { posterior choroidal artery }\end{array}$ \\
\hline Medial inferior pontine & $\begin{array}{l}\text { Paramedian pontine reticular } \\
\text { formation; CN VI nucleus or } \\
\text { fibers; middle cerebellar peduncle; } \\
\text { corticospinal tract; medial } \\
\text { lemniscus }\end{array}$ & $\begin{array}{l}\text { Ipsilateral CN VI or horizontal gaze } \\
\text { palsy; ataxia. Paresis and } \\
\text { impaired lemniscal sensation of } \\
\text { contralateral limbs }\end{array}$ & $\begin{array}{l}\text { Due to occlusion of paramedian } \\
\text { perforating vessel }\end{array}$ \\
\hline $\begin{array}{l}\text { Lateral inferior pontine } \\
\text { (AICA syndrome) }\end{array}$ & $\begin{array}{l}\text { Cranial nerve VII nucleus or fibers; } \\
\text { middle cerebellar peduncle; inferior } \\
\text { cerebellar peduncle; corticospinal } \\
\text { tract; principle and spinal nucleus } \\
\text { of CN V; lateral spinothalamic tract; } \\
\text { solitary tract; flocculus and inferior } \\
\text { surface of cerebellar hemisphere }\end{array}$ & $\begin{array}{l}\text { Ipsilateral cerebellar ataxia; loss of } \\
\text { pain and temperature sensation } \\
\text { and diminished light touch sensation } \\
\text { of face; impaired taste sensation; } \\
\text { central Horner's syndrome; } \\
\text { deafness; peripheral type of facial } \\
\text { palsy. Loss of pain and temperature } \\
\text { sensation of contralateral limbs }\end{array}$ & $\begin{array}{l}\text { Due to occlusion of anterior inferior } \\
\text { cerebellar artery }\end{array}$ \\
\hline Medial midpontine & $\begin{array}{l}\text { Middle cerebellar peduncle; } \\
\text { corticospinal tract; medial } \\
\text { lemniscus }\end{array}$ & $\begin{array}{l}\text { Ipsilateral ataxia. Contralateral weakness } \\
\text { of arm, leg, and face; gaze deviation; } \\
\pm \text { impaired lemniscal sensation }\end{array}$ & $\begin{array}{l}\text { Due to occlusion of paramedian } \\
\text { perforating vessel }\end{array}$ \\
\hline Lateral midpontine & $\begin{array}{l}\text { Middle cerebellar peduncle; CN V } \\
\text { motor and sensory nuclei or fibers }\end{array}$ & $\begin{array}{l}\text { Ipsilateral ataxia; weakness of muscles } \\
\text { of mastication; impaired facial } \\
\text { sensation }\end{array}$ & $\begin{array}{l}\text { Due to occlusion of short } \\
\text { circumferential artery }\end{array}$ \\
\hline $\begin{array}{l}\text { Medial superior } \\
\text { pontine }\end{array}$ & $\begin{array}{l}\text { Superior cerebellar peduncle and/or } \\
\text { middle cerebellar peduncle; medial } \\
\text { longitudinal fasciculus; central } \\
\text { tegmental tract; corticospinal } \\
\text { tract; medial lemniscus }\end{array}$ & $\begin{array}{l}\text { Ipsilateral ataxia; internuclear } \\
\text { ophthalmoplegia. Contralateral } \\
\text { weakness of arm, leg, and face; } \\
\pm \text { impaired lemniscal sensation. } \\
\text { Palatal myoclonus }\end{array}$ & $\begin{array}{l}\text { Due to occlusion of paramedian } \\
\text { perforating vessel }\end{array}$ \\
\hline $\begin{array}{l}\text { Medial medullary } \\
\text { syndrome }\end{array}$ & $\begin{array}{l}\text { XII nucleus or fibers; medullary } \\
\text { pyramid(at/near decussation); } \\
\pm \text { medial lemniscus }\end{array}$ & $\begin{array}{l}\text { Ipsilateral tongue weakness; contralateral } \\
\text { hemiparesis (sparing the face); } \\
\pm \text { impairment of posterior column } \\
\text { function; lateral spinothalamic } \\
\text { functions spared }\end{array}$ & $\begin{array}{l}\text { Due to ischemia in the distribution of } \\
\text { the paramedian perforator or the } \\
\text { anterior spinal artery, findings may } \\
\text { be bilateral and of variable laterality } \\
\text { due to involvement of the pyramidal } \\
\text { decussation and variations in the } \\
\text { anatomy of the anterior spinal artery }\end{array}$ \\
\hline $\begin{array}{l}\text { Lateral medullary } \\
\text { syndrome }\end{array}$ & $\begin{array}{l}\text { Spinal tract of CN V and its nucleus; } \\
\text { nucleus ambiguous; emerging fibers } \\
\text { of CNs IX and X; LST; descending } \\
\text { sympathetic fibers; vestibular nuclei; } \\
\text { inferior cerebellar peduncle; afferent } \\
\text { spinocerebellar tracts; lateral } \\
\text { cuneate nucleus }\end{array}$ & $\begin{array}{l}\text { Loss of pain and temperature ipsilateral } \\
\text { face and contralateral body; decreased } \\
\text { ipsilateral corneal reflex; weakness of } \\
\text { ipsilateral soft palate; loss of ipsilateral } \\
\text { gag reflex; paralysis of ipsilateral vocal } \\
\text { cord; ipsilateral central Horner's } \\
\text { syndrome; nystagmus; cerebellar ataxia } \\
\text { of ipsilateral limbs; lateropulsion }\end{array}$ & $\begin{array}{l}\text { Due to ischemia in posterior inferior } \\
\text { cerebellar artery distribution, but } \\
\text { more often due to vertebral artery } \\
\text { occlusion }\end{array}$ \\
\hline
\end{tabular}

Adapted from Campbell. ${ }^{1}$

AICA, anterior inferior cerebellar artery; CN, cranial nerve; SCA, spinocerebellar ataxia.

central pontine myelinolysis, Arnold-Chiari malformations, and syringobulbia. Considering that the brainstem is such a compact structure, with cranial nerve nuclei, nerve fascicles, and long ascending and descending tracts all closely juxtaposed, a plethora of clinical findings are usually present to help in localization. Most brainstem cases with involvement of multiple cranial nerves will have telltale clues such as long tract signs, gaze palsies, internuclear ophthalmoplegia, and complex spontaneous eye movement abnormalities. Focal brainstem lesions are often characterized by "crossed" syndromes, consisting of ipsilateral cranial nerve dysfunction and contralateral long motor or sensory tract dysfunction. Due to the rich vestibular and cerebellar connections, patients with 
brainstem disease will often complain of vertigo, gait unsteadiness, ataxia, discoordination, nausea, and vomiting. Thus, a history aimed at eliciting these symptoms as well as a careful complete neurologic exam looking for these associated signs is necessary to aid in localizing a process to the brainstem.

\section{MULTIPLE CRANIAL NEUROPATHIES: OVERVIEW}

There are 12 pairs of cranial nerves that innervate most of the structures of the head and neck. The afferent and efferent connections of these 12 cranial nerves traverse the meninges, subarachnoid space, bony structures of the skull, and superficial soft tissues. Dysfunction of these nerves may occur at any of these sites along their course. Therefore, it is not surprising that a large number of pathologic processes initially are manifested by cranial nerve dysfunction. ${ }^{3}$ These disease processes may involve homologous nerves on the two sides (i.e., bilateral facial palsy) or different nerves on the same or contralateral side. In some conditions, a group of nerves is involved in a discreet anatomic region. The progression of cranial nerve dysfunction may follow some anatomical pattern or appear randomly. Multiple cranial nerves may be affected from the outset, or the process may begin with one nerve with subsequent involvement of others. The presence or absence of pain may also provide a clue to the diagnosis.

The majority of the literature regarding etiologies of multiple cranial neuropathies consists of case reports or small case series with very few reported large series. The largest reported series to date of multiple cranial neuropathies was that of Keane. ${ }^{2}$ In his review, Keane reported the findings of 979 cases of simultaneous or serial involvement of two or more different cranial nerves. Of note, in his review, the first and ninth cranial nerves were not examined in a systemic fashion, and therefore, were not tabulated. Although patients with botulism were included, those with a diagnosis of myasthenia gravis were excluded. According to Keane's series, the abducens nerve was the most commonly involved cranial nerve, followed by the facial nerve. The oculomotor and trigeminal nerves were the third and fourth most affected. Oculomotor and trochlear dysfunction was the most common combination of cranial nerve dysfunction followed closely by trigeminal plus abducens as well as trigeminal plus facial nerve palsies. An exhaustive list of conditions listed by etiologies resulting in multiple cranial neuropathies can be seen in Table 2 . Given the extensive differential diagnosis associated with multiple cranial nerve palsies, one must develop a systematic way to approach these patients. As always, this approach should be guided by the localization. We will first consider meningeal processes causing meningitis followed by neoplastic processes affecting the clivus/ skull base. Next, a brief discussion of extramedullary vascular etiologies, bone disorders, and traumatic considerations as well as peripheral nerve considerations will ensue. Finally, disorders of specific cranial nerve groups/ syndromes will be discussed.

\section{CHRONIC MENINGITIS}

\section{Infectious Meningitis}

Chronic meningitis is always in the differential diagnosis of multiple cranial neuropathies. Chronic meningitis usually presents in a subacute or insidious manner. Common presenting symptoms include insidious headache, fever, and neck stiffness; however, cranial nerve abnormalities are not an uncommon presentation. The neurologic signs and symptoms of chronic meningitis are often nonspecific, and the pursuit of this diagnosis often requires an intensive investigation of potential exposures and associated systemic symptoms. Furthermore, the number of recognized etiologies of chronic meningitis is also increasing, adding further to the complexity of this evaluation. ${ }^{4}$ In turn, the diagnostic investigation often depends on a detailed historical probe for the exposures and features of systemic disease, which may provide essential clues allowing for a more directed approach to laboratory testing. ${ }^{5}$ A careful history should be obtained with attention to recent travel history, exposure to animals, contacts with similar symptoms, and the patient's immune status. A meticulous general physical examination is necessary including examination of lungs, eyes, liver, joints, and skin, which again may reveal signs suggestive of a systemic illness. As mentioned, the list of potential etiologies for chronic meningitis is broad and includes infectious, inflammatory, and neoplastic processes. In Keane's series, infectious etiologies comprised $10 \%$ of their cases with 48 of 102 cases secondary to meningitis. ${ }^{2}$ These infectious causes can be further classified into bacterial, viral, fungal, and parasitic etiologies. Because so many causes of chronic meningitis exist, this article will focus on a few that have been more closely associated with multiple cranial neuropathies.

Lyme disease is associated with an array of neurologic complications involving both the peripheral and central nervous system (CNS). Neurologic involvement is seen in $\sim 15 \%$ of infected individuals, most commonly during the second stage of infection, often several weeks following inoculation. ${ }^{5}$ Facial nerve palsies are a frequent neurologic complication of Lyme disease, occurring as early as 30 days after the classic erythema chronicum migrans rash, and may be associated with an aseptic meningitis and painful radiculitis. Although facial nerve palsies are the most cited cranial nerve abnormality, involvement of cranial nerves (CNs) II, V, and VIII have also been reported. ${ }^{6}$ Although not as common in 
Table 2 Differential Diagnosis of Extramedullary Etiologies of Multiple Cranial Neuropathies

\begin{tabular}{|c|c|c|c|}
\hline \multicolumn{4}{|l|}{ Infection } \\
\hline Bacterial & Fungal & Viruses & Parasites \\
\hline Lyme disease & Cryptococcosis & Herpes Zoster & Chagas disease \\
\hline Syphilis & Histoplasmosis & Epstein-Barr & Cysticercosis \\
\hline Pseudomonas sp. & Coccidiomycosis & Cytomegalovirus & \\
\hline Mycoplasma & Blastomycosis & HIV $1 \& 2$ & \\
\hline Mycobacterium tuberculosis & Mucormycosis & & \\
\hline \multirow[t]{2}{*}{ Bacterial sinusitis } & Aspergillosis & & \\
\hline & Candida species & & \\
\hline \multicolumn{4}{|l|}{ Inflammatory diseases } \\
\hline Sarcoidosis & Vasculitis & & \\
\hline Behçet's disease & Wegener's granulomatosis & & \\
\hline Idiopathic hypertrophic cranial pachymeningitis & Lymphomatoid granulomatosis & & \\
\hline AIDP/Guillain-Barre/Miller-Fisher syndrome & Polyarteritis nodosa & & \\
\hline Amyloidosis & Temporal arteritis & & \\
\hline Idiopathic cranial polyneuropathy & Connective tissue disease & & \\
\hline \multirow[t]{4}{*}{ Tolosa-Hunt syndrome } & Rheumatoid arthritis & & \\
\hline & Sjögren's disease & & \\
\hline & Systemic lupus erythematosus & & \\
\hline & Scleroderma & & \\
\hline \multicolumn{4}{|l|}{ Neoplastic } \\
\hline Carcinomatous meningitis & Giant cell tumor & Meningioma & \\
\hline Metastases (solid tumors) & Hemangioblastoma & Plasmacytoma & \\
\hline Lymphoma & Dermoid/epidermoid & Cholesteatoma & \\
\hline Leukemia & Adenoid cystic carcinoma & Craniopharyngioma & \\
\hline Primary leptomeningeal & Mucoepidermoid carcinoma & Chordoma & \\
\hline Gliomatosis & Glomus tumor & Fibrosarcoma & \\
\hline Nasopharyngeal carcinoma & Schwannoma & Rhabdomyosarcoma & \\
\hline Metastatic skull-based tumor & Pituitary adenoma/apoplexy & Histiocytosis & \\
\hline \multicolumn{4}{|l|}{ Primitive neuroectodermal tumor } \\
\hline \multicolumn{4}{|l|}{ Neurinoma } \\
\hline \multicolumn{4}{|l|}{ Vascular } \\
\hline Vertebrobasilar dolichoectasia & Carotid-cavernous fistula & Diabetes & \\
\hline Aneurysm & Carotid artery dissection & Sickle-cell disease & \\
\hline \multicolumn{4}{|l|}{ Bone disease } \\
\hline \multicolumn{4}{|l|}{ Osteopetrosis } \\
\hline \multicolumn{4}{|l|}{ Paget's disease } \\
\hline \multicolumn{4}{|l|}{ Hyperostosis cranialis interna } \\
\hline \multicolumn{4}{|l|}{ Fibrous dysplasia } \\
\hline \multicolumn{4}{|l|}{ Toxic } \\
\hline \multicolumn{4}{|c|}{ Chemotherapy induced neuropathies (vincristine/vinblastine/cisplatinum) } \\
\hline \multicolumn{4}{|l|}{ Trauma } \\
\hline \multicolumn{4}{|l|}{ Closed head injury } \\
\hline Carotid endarterectomy & & & \\
\hline
\end{tabular}

AIDP, acute inflammatory demyelinating polyneuropathy; HIV, human immunodeficiency virus.

the United States, tuberculosis remains, from a global standpoint, one of the more common infectious agents associated with chronic meningitis. It is estimated that about one third of the world's population has been infected with tuberculosis. ${ }^{4}$ In one series of patients with chronic meningitis without a known predisposing cause, $40 \%$ were found to have tuberculous meningitis. As is common with any meningitis, tuberculous menin- gitis most often presents with headache, fever, vomiting, photophobia, ${ }^{8}$ and encephalopathy ${ }^{9}$; however, cranial nerve palsies are reported in up to $19 \%{ }^{10}$ The sixth nerve is usually involved first and is most frequently affected. ${ }^{3}$ Although neurosyphilis is rare today, it still must be considered as a cause of multiple cranial neuropathies. Cranial neuropathies are seen in about one third. ${ }^{5}$ Less commonly reported bacterial infections 
associated with multiple cranial neuropathies include Mycoplasma and Pseudomonas aeruginosa. ${ }^{11,12}$

CNS fungal infections, although rare, have increased due to the increased prevalence of immunosuppression from acquired immunodeficiency syndrome (AIDS), organ transplantation, corticosteroid use, and chemotherapy. The most common fungal pathogen is Cryptococcus neoformans, which accounts for more than half of all CNS fungal meningitis cases. ${ }^{4}$ Other frequent fungal infections include Coccidiodes immitis, histoplasmosis, and blastomycosis. In those at risk for opportunistic infections, Aspergillus sp., mucormycosis (Zygomycetes species), and Candida species must also be considered, often from extension to the orbit from the sinuses.

Viruses are a rare cause of chronic meningitis, with the exception of human immunodeficiency virus (HIV), which often causes a persistent meningitis. ${ }^{13}$ HIV-1 has had a known association with cranial neuropathies; however, more recently HIV-2 has also been shown to present with multiple cranial nerve palsies. ${ }^{14}$ Other viruses to consider include herpes zoster, ${ }^{15}$ Epstein-Barr, ${ }^{16}$ and cytomegalovirus. ${ }^{17}$

Meningeal parasitic infections leading to cranial neuropathies are rare, with the exception of cysticercosis, which is common in Mexico, Central America, and South America. In Keane's series, six cases of multiple cranial neuropathies were secondary to cysticercosis. ${ }^{2}$ Although more commonly characterized by cardiac involvement in Central and South America, Chaga's disease (Trypanosoma cruzi) has been associated with cranial neuropathies in a patient with AIDS. ${ }^{18}$

\section{Noninfectious Inflammatory Meningitis}

Other noninfectious, inflammatory processes that may also present with a chronic meningitis causing multiple cranial nerve palsies include granulomatous and vasculitic processes. Of the granulomatous diseases, neurosarcoidosis occurs worldwide with a peak incidence between 20 and 40 years. Neurosarcoidosis develops in $\sim 5$ to $15 \%$ of patients with systemic sarcoidosis ${ }^{19}$ with as many as $50 \%$ presenting with neurologic symptoms at the time of diagnosis. ${ }^{20,21}$ Bilateral facial nerve palsies is a common presentation, occurring in $5 \%$ of patients with sarcoidosis. ${ }^{22}$ Although the neurologic manifestations of neurosarcoidosis are diverse, $~ 50$ to $75 \%$ of patients will develop cranial nerve palsies that are often multiple. The nerves most commonly involved are VII, II, IX, X, and VIII. ${ }^{4}$ Another multiorgan granulomatous disease that can cause dysfunction of multiple cranial nerves is Behçet's syndrome. This syndrome is more common in the Mediterranean, East Asian, and Middle Eastern regions with a constellation of oral aphthous ulcers, genital ulcers, and uveitis. Neurologic involvement has been reported in 3 to $20 \%$ of cases. ${ }^{23}$ Multiple cranial nerves may be involved as a result of meningeal involvement as well as from brainstem involvement. CNs II and VIII are most commonly affected. ${ }^{24}$

Systemic vasculitides have varied neurologic complications. Several forms of vasculitis deserve special mention due to their association with multiple cranial neuropathies. Wegener's granulomatosis is the one with the greatest tendency to affect multiple cranial nerves. In a review of 324 consecutive patients seen at the Mayo Clinic, 33.6\% had documented neurologic involvement with mononeuritis multiplex; cranial neuropathies were the most common neurologic findings. ${ }^{25}$ In another review, the CNS was affected in only $8 \%$ of patients. ${ }^{26}$ CNs II, VI, VII, and VIII were most often affected. Renal involvement is frequently present in patients who have neurologic manifestations. ${ }^{27}$ Granulomatous masses may also extend from the nasal and paranasal sinuses causing a restrictive ophthalmoplegia simulating cranial nerve palsies. Another systemic granulomatous vasculitis with a predilection for the CNS is lymphomatoid granulomatosis, a malignant lymphoreticular disorder that usually presents with constitutional symptoms and skin lesions resembling erythema nodosum. ${ }^{28}$ Pulmonary involvement is characteristic and the diagnosis is unlikely in the absence of pulmonary lesions. ${ }^{27}$ Neurologic manifestations occur in 30\%, with cranial neuropathies in $11 \% .{ }^{29}$ Polyarteritis nodosa is a systemic necrotizing vasculitis with multiorgan involvement. Although not as common as in Wegener's granulomatosis, cranial nerve involvement occurs, with CNs III and VIII most commonly involved. ${ }^{4}$ One other vasculitic process, temporal arteritis, deserves brief mention. Anterior ischemic optic neuropathy and ophthalmoplegia from involvement of the extraocular muscles are common, but other cranial nerves are usually spared. ${ }^{3}$

Several connective tissue disorders associated with vasculitis must also be considered. In particular, rheumatoid arthritis, Sjögren's syndrome, scleroderma, and systemic lupus erythematosus have known associations with cranial neuropathies. ${ }^{30-33}$ A full discussion of the neurologic manifestations of the connective tissue diseases are beyond the scope of this discussion, and the reader is referred to the cited references for a detailed review. Another inflammatory condition with cranial nerve involvement is idiopathic hypertrophic cranial pachymeningitis. This is a chronic fibrosing inflammatory condition of the dura mater resulting in thickening of the dura. ${ }^{34}$ The clinical presentation is usually nonspecific with signs and symptoms of increased intracranial pressure (ICP), including headache, nausea and vomiting, as well as ataxia and focal neurologic complaints. Variable involvement of cranial nerves V-XII may be present. ${ }^{35} \mathrm{~A}$ final inflammatory consideration in patients presenting with multiple cranial neuropathies is amyloidosis. Although certain familial forms of amyloidosis, particularly type IV, commonly produce cranial 
neuropathies, ${ }^{36}$ primary amyloidosis less commonly presents in this fashion. ${ }^{37}$

\section{Neoplastic Meningitis}

Neoplastic processes are an important cause of multiple cranial neuropathies, especially when the patient presents subacutely (days to weeks) in the absence of pain. Patients with neoplastic meningitis usually have accompanying headache, meningeal signs, and evidence of increased ICP. Important causes of neoplastic meningitis include carcinomatous and lymphomatous meningitis. Neoplastic meningitis is diagnosed in up to $15 \%$ of patients with systemic carcinomas or hematologic malignancies, and may be the first presentation in 5 to $10 \%$ of patients. Small cell lung cancers, melanomas, and myeloblastic leukemias are the most likely tumors to spread to the meninges. ${ }^{38,39}$ Although breast cancer has a low predilection for spread to the meninges, it is one of the most common tumors to cause neoplastic meningitis because of its overall high frequency. ${ }^{40}$ Other tumors that may have meningeal involvement are lymphomas, gastrointestinal cancers, other lung cancers, and primary brain tumors including medulloblastoma, ependymoma, and cerebellar astrocytomas. ${ }^{38,39}$ Whereas leptomeningeal metastatic disease from solid tumors is more likely to present with spinal cord or radicular involvement, diffuse meningeal involvement from hematologic malignancies is more likely to present with multiple cranial nerve palsies. Lymphomatous meningitis may result from systemic or primary CNS lymphoma. Primary leptomeningeal lymphoma has rarely been described. ${ }^{41}$ Primary leptomeningeal gliomatosis is a rare form of cancer that arises in the absence of a known primary CNS neoplasm and may present with multiple cranial nerve palsies. ${ }^{42}$

\section{NEOPLASMS AFFECTING THE CLIVUS/SKULL BASE}

In addition to neoplastic processes affecting the meninges, other neoplasms occurring at the base of the skull may present with multiple cranial nerve palsies. In Keane's series, tumor was identified as the cause of multiple cranial neuropathy in $30 \%$ of the patients, representing the most common group of etiologies. ${ }^{2}$ In terms of localization, there was a clival/skull based localization in $13 \%$ of patients. Nasopharyngeal carcinoma occurs more often in younger patients than do other head and neck cancers. This tumor may be associated with an Epstein-Barr viral infection. It can spread by extension to the skull base. It may infiltrate the pterygopalatine fossa and the maxillary nerve and may even spread to the cavernous sinus. About 20\% of patients have cranial nerve involvement at the time of its diagnosis. ${ }^{1} \mathrm{CN}$ VI is most often involved, and in fact, is often the presenting manifestation. Involvement of
CNs II and $\mathrm{V}$ is also common whereas facial nerve palsies are uncommon. CN XII may be affected in advanced cases with extensive involvement of the skull base. Additionally, radiotherapy for this tumor may itself cause cranial neuropathies, especially of CN XII. At times, nasopharyngeal carcinomas may also even erode through the clivus. A combination of $\mathrm{CN}$ VI and XII palsies is particularly suggestive of a neoplastic process involving the clivus. ${ }^{1}$ Another tumor that commonly involves the clivus and often presents with multiple cranial neuropathies is a chordoma, a rare primary bone tumor derived from the remnants of the primitive notochord that usually presents in men in the sixth decade. Although histologically benign, with posterior extension it may become locally invasive causing cranial nerve dysfunction and even brainstem compression. Other skull-based neoplasms that may present in a similar fashion include metastasis, meningioma, lymphoma, myeloma, histiocytosis, neurinoma, giant cell tumor, hemangioblastoma, and various primary bone tumors. Prepontine neoplasms, such as exophytic gliomas, dermoid, and epidermoid tumors often present with multiple cranial neuropathies, particularly $\mathrm{CN}$ III, V, and VI dysfunction. Neoplastic involvement of the temporal bone often presents with facial nerve palsies and involves the lower cranial nerves by direct extension. Such neoplasms involving the temporal bone or external auditory canal include adenoid cystic carcinoma, adenocarcinoma, and mucoepidermoid carcinoma. These are often associated with chronic otitis media and otalgia. Other neoplasms reported in Keane's series not discussed above include schwannomas, pituitary adenomas, intramedullary brainstem gliomas and ependymomas, fibrosarcomas, rhabdomyosarcomas, primitive neuroectodermal tumors, leukemias, craniopharyngiomas, cholesteatomas, and glomus jugulare tumors. ${ }^{2}$ Some of these will be discussed further below in the appropriate cranial nerve groups.

\section{EXTRAMEDULLARY VASCULAR DISORDERS}

Brainstem vascular syndromes may cause multiple cranial neuropathies due to ischemia. In addition, extramedullary vascular disease must also be considered in the differential diagnosis. Vertebrobasilar dolichoectasia may compress multiple cranial nerves with CNs III, VI, and $\mathrm{V}$ most commonly involved. Patients with a tortuous basilar artery of normal caliber are more likely to have isolated cranial neuropathies; those with basilar artery ectasia or with a giant, fusiform aneurysm are more likely to have multiple cranial neuropathies. ${ }^{1}$ Cranial nerve palsies occasionally occur in carotid artery dissection. Ipsilateral headache, Horner's syndrome, and lower cranial neuropathies may suggest carotid dissection, even in the absence of ischemic symptoms. CN XII 
is invariably involved and in some patients, other cranial nerves may also be affected. The etiology of lower cranial neuropathy in carotid dissection is unclear, but may be related to compression, to stretching by the aneurysmal dilatation, or to ischemia of the segmental arteries supplying the nerves, particularly the ascending pharyngeal artery. ${ }^{43}$ Although diabetes often causes isolated cranial neuropathies, only rarely does it affect more than one cranial nerve at a time. ${ }^{44}$ Finally, sickle cell disease has rarely been reported as a cause of multiple cranial neuropathies, mainly CNs V and VII. ${ }^{45}$

\section{BONE DISORDERS}

Although less common in comparison to many of the above considerations, disorders of bone can also result in compressive cranial neuropathies. Osteopetrosis (Albers-Schonberg or marble bone disease) is a rare congenital bone disorder characterized by defective osteoclastic bone resorption. Subsequently, a generalized increase in bone density occurs and the cranial foramina can become narrowed, resulting in multiple cranial neuropathies. ${ }^{46}$ Paget's disease of the bone is characterized by increased bone remodeling, bone hypertrophy, and abnormal bone structure that can lead to bone deformity and multiple cranial nerve entrapment. ${ }^{47}$ Fibrous dysplasia of the cranium is another entity in which normal bone is replaced by abnormal fibroconnective tissue proliferation. Multiple cranial bones can be affected as well as the clivus/skull base, and the pattern of neurologic abnormalities depends on the pattern of cranial bone involvement. Expansive lesions of the jugular foramen may encroach on and cause dysfunction of the lower cranial nerves. A less well-understood inherited bone disorder with reported involvement of multiple cranial nerves is hyperostosis cranialis interna. This autosomal dominant condition is characterized by hyperostosis and osteosclerosis of the calvaria and base of the skull with initial manifestations of recurrent facial nerve (CN VII) palsies with variable impairment of smell $(\mathrm{CN} \mathrm{I})$, vision (CN II), and hearing (CN VIII). ${ }^{48}$ The age of onset ranges from 9 to 32 years. Curiously, the hyperostosis is confined to the skull, with no long bone involvement.

\section{TRAUMA}

Blunt and penetrating head injuries are important considerations. In Keane's series, trauma was one of the more common causes of multiple cranial neuropathies representing $12 \%$ of the cases, similar in frequency to vascular etiologies. In terms of blunt trauma, automobile and motorcycle accidents were most common followed by falls and beatings. ${ }^{2}$ Penetrating injury was less than half as common with gunshot wounds being the predominant cause. Although accidental trauma represented a large portion of traumatic etiologies, iatrogenic causes must also be considered in this context. For example, cranial neuropathies, sometimes multiple, are a well-established complication of carotid endarterectomies, posterior triangle lymph node biopsies, and other surgical procedures on the head and neck, especially radical procedures.

\section{DISORDERS OF CRANIAL NERVE GROUPS}

In some locations, two or more cranial nerves are bundled in a common anatomic space, and when a focal disease process occurs, the entire cluster of nerves may be involved. Most often, disorders of cranial nerve groups are due to mass effect and are commonly secondary to neoplasms. As with the vascular brainstem syndromes, many of these carry eponyms. Many of these syndromes are rare, however, in neurologic practice. Familiarity with the more common of these syndromes may aid in localization when the relevant cranial nerves are involved. Relatively common anatomical syndromes involve the cavernous sinus, the cerebellopontine angle, and the jugular foramen.

\section{Cavernous Sinus Syndrome}

The cavernous sinuses are paired venous channels that lie on either side of the sphenoid bone and sella turcica, lateral to the pituitary. They extend from the superior orbital fissure to the apex of the petrous temporal bone. The two sides are connected by an anterior and posterior inter cavernous sinus. The internal carotid artery with its pericarotid sympathetic plexus runs through the sinus whereas the oculomotor, trochlear, abducens, and trigeminal nerves ( $1^{\text {st }}$ and $2^{\text {nd }}$ division) pass laterally on its wall. Common signs and symptoms of cavernous sinus disease include ophthalmoplegia, orbital congestion, chemosis, periorbital edema, proptosis, and facial sensory loss as well as Horner's syndrome from involvement of the sympathetics. According to Keane's series, the cavernous sinus was the most frequent site of multiple cranial nerve involvement representing $25 \%$ of cases. ${ }^{2}$ Common causes can be divided into vascular, neoplastic, inflammatory, and miscellaneous disorders. In severe cases, all of the nerves passing through the sinus may be involved; however, isolated involvement of individual nerves also occurs, especially the abducens nerve. A Horner's syndrome in conjunction with an abducens palsy is particularly localizing.

Of the vascular causes, carotid aneurysms, carotid cavernous fistulas, and thrombosis need to be considered. If large enough, intracavernous aneurysms may compress and distort the contents of the cavernous sinus, often resulting in an indolent painful ophthalmoplegia. Intracavernous aneurysms do not have a significant risk of subarachnoid hemorrhage. When rupture does occur, it tends to remain local and may result in formation of a 
cavernous-carotid fistula. ${ }^{49}$ A carotid cavernous fistula is a communication between the carotid artery and the cavernous sinus. These may be further classified into direct or indirect fistulas. In direct fistulas, the cavernous carotid artery and cavernous sinus are in direct continuity. These often develop spontaneously from a ruptured cavernous aneurysm as above, or may be secondary to trauma, such as a closed head injury. They are characterized by the classic triad of chemosis, pulsatile exophthalmos, and an audible bruit over the eye on auscultation. These often require complex interventional management. ${ }^{49}$ In indirect fistulas, shunts are established through meningeal branches from the carotid system. These tend to be more insidious with arterialization of the conjunctival vessels without an audible bruit and often resolve spontaneously. Cavernous sinus thrombosis usually results from paranasal sinus infections, orbital cellulitis, or a facial infection (such as a furuncle). Staphylococcus is the most common causative organism; however, pneumococcal and fungal infections should also be considered, particularly in immunocompromised individuals and diabetics. In diabetics, mucormycosis is of particular concern.

Tumors are the most common cause of cavernous sinus syndrome. ${ }^{49}$ These may be metastatic disease, a result of local tumor extension (nasopharyngeal carcinoma, pituitary adenoma or craniopharyngioma) or a primary tumor (meningioma, lymphoma). Finally, the cavernous sinus syndrome can result from any inflammatory granulomatous processes such as sarcoidosis, Wegener's granulomatosis, or polyarteritis nodosa. Another inflammatory disorder that must be considered is Tolosa-Hunt syndrome. This is an idiopathic inflammatory granulomatous disorder that typically presents with painful ophthalmoplegia. Once mass lesion has been excluded, it is the most common cause of cavernous sinus syndrome. Although spontaneous remission occurs in up to a third of patients, the universally positive response to steroids is often regarded as a diagnostic criteria. ${ }^{49}$ One must consider, however, that other processes, such as tumors (especially lymphoma) may also show an initial response to steroids.

\section{Cerebellopontine Angle}

The boundaries of the cerebellopontine angle include the inferior surface of the cerebellar hemisphere, the lateral aspect of the pons, and the superior surface of the inner third of the petrous ridge. It spans longitudinally from $\mathrm{CN} \mathrm{V}$ through $\mathrm{CN} \mathrm{X}$. Lesions of the cerebellopontine angle are invariably neoplasms, most of which are benign. Vestibular schwannomas are by far the most common tumor, arising from the vestibular portion of CN VIII within the internal auditory canal. Less common neoplasms include meningiomas, epidermoids, and much less commonly metastases and cholesteatomas.
The initial symptoms are usually progressive sensorineural hearing loss and tinnitus. Because of the slowgrowing nature and the ability of the vestibular system to compensate, frank vertigo is unusual; however, gait dysequilibrium is not uncommon. As the mass expands, cranial nerve dysfunction ensues accompanied by dysfunction of CN VII causing a lower motor neuron facial paresis without hyperacusis. CN V dysfunction causing facial sensory loss is also common. CNs VI, IX and X are less commonly involved, usually later in the course. If the lesion continues to grow, pressure on the cerebellum or its peduncles result in ipsilateral ataxia and incoordination. Nystagmus and gaze palsies may result from pontine compression.

\section{Lower Cranial Nerve Syndromes}

The lower cranial nerve syndromes involve CNs IX-XII unilaterally in various combinations. These nerves exit the skull just above the foramen magnum. CNs IX-XI exit through the jugular foramen along with the jugular vein. CN XII exits through the hypoglossal canal just inferiorly. The symptoms of lower cranial nerve disease, including dysphagia, dysphonia, and dysarthria are common reasons for neurologic consultation. Therefore, a knowledge of the cranial foramina and their contents, as well as the relationship of structures near the skull base, are essential for the neurologist who will invariably encounter one of the many diseases that can affect this area.

Jugular foramen syndrome, or Vernet's syndrome, is the prototype lower cranial nerve syndrome, characterized by ipsilateral paralysis of CNs IX, X, and XI. This syndrome is caused by a lesion at the jugular foramen or in the retroparotid space. Glomus tumors (paragangliomas) are common causes of jugular foramen syndrome. They are benign, usually spontaneous, slowgrowing head and neck tumors that are thought to arise from widely distributed paraganglionic tissue that originates from the neural crest cells. Glomus tumors commonly arise in the jugular bulb (glomus jugulare), the middle ear (glomus tympanicum), and the nodose ganglion of the vagus nerve (glomus vagale). Although slow growing, they may erode through bone and extend into the jugular foramen or even into the hypoglossal canal. Other common inciting lesions are schwannomas, meningiomas, and metastases. Rarer causes include retroparotid abscesses, chordomas, and thrombosis of the jugular bulb. The term jugular foramen syndrome is often used to refer to any combination of palsies affecting the lower four cranial nerves; however, several other eponymic syndromes deserve mention. Collet-Sicard syndrome, or intercondylar space syndrome, consists of jugular foramen syndrome (dysfunction of CNs IX, X and XI) with additional involvement of CN XII. Villaret's syndrome is Collet-Sicard plus the addition of sympathetic 
involvement (Horner's syndrome). This is also referred to as the retropharyngeal space syndrome. If the inciting process extends into the retroparotid space, there may be additional CN VII involvement. The same etiologic considerations apply for all these syndromes; therefore, from a practical standpoint, considering all collectively as jugular foramen syndromes stands to reason. Finally, although not technically a lower cranial nerve syndrome, the petrous apex syndrome can progress to include the lower cranial nerves. Otherwise known as Gradenigo's syndrome, this syndrome is typically associated with suppurative otitis media affecting the petrous apex of the temporal bone. It typically presents with pain in a trigeminal nerve distribution combined with abducens palsy. If the infection spreads to the skull base, then features of jugular foramen syndrome may coexist.

\section{PERIPHERAL NERVOUS SYSTEM CONSIDERATIONS}

Other conditions that may present with prominent weakness of bulbar muscles and mimic brainstem dysfunction include neuromuscular junction disorders, peripheral neuropathies, and certain myopathies. See Table 3 for a list of peripheral nervous system disorders that can present with bulbar dysfunction. Peripheral nerve localization represented $17 \%$ of the cases in Keane's series, dominated by cases of Guillain-Barre syndrome and Miller-Fisher syndrome. ${ }^{2}$ Polyneuritis cranialis is a multiple cranial neuropathy that has been attributed to Lyme disease, herpes zoster, and as a Guillain-Barre variant. ${ }^{50}$ Other causes of polyneuropathy that commonly have cranial nerve involvement include diphtheria, HIV, Lyme disease, sarcoidosis, and certain chemotherapeutic agents. Idiopathic cranial polyneuropathy is another

\section{Table 3 Peripheral Nervous System Considerations}

Polyneuropathies with cranial nerve involvement
Guillain-Barre syndrome and its variants
Diabetes
Diphtheria
Human immunodeficiency virus/acquired immunodeficiency
syndrome
Lyme disease
Sarcoidosis
Idiopathic cranial polyneuropathy
Chemotherapy-related neuropathies (vincristine/vinblastine/
cisplatinum)
Neuromuscular junction disease
Myasthenia gravis
Botulism
Myopathies with bulbar involvement
Mitochondrial (chronic progressive external ophthalmoplegia)
Fascioscapular humeral dystrophy (FSHD)
Oculopharyngeal dystrophy

specific peripheral nerve entity, which has been well characterized. This syndrome often presents with constant, usually retroorbital, facial pain followed suddenly by cranial nerve palsies. The cranial nerves most commonly involved are the oculomotor, trochlear, and abducens, followed by the trigeminal and facial nerves. The lower cranial nerves may also be involved. ${ }^{11}$ The etiology remains unknown, and some have speculated an overlap with Tolosa-Hunt syndrome, especially considering the mutual response to steroids. Although they can occasionally present with bulbar dysfunction, neuromuscular junction disorders and myopathies can usually be distinguished by clinical history.

\section{DIAGNOSIS}

The diagnostic possibilities for multiple cranial nerve palsies are legion, and the diagnosis remains a formidable challenge despite the advances of modern medicine. Although the list of possibilities is exhaustive, many causes can be eliminated by radiologic and cerebrospinal fluid (CSF) studies. The initial evaluation should include magnetic resonance imaging (MRI) of the brain with gadolinium; measurement of erythrocyte sedimentation rate and $\mathrm{C}$ reactive protein; blood counts; routine blood chemistry tests; and studies of CSF, including cultures and cytology. The main objective of routine neuroimaging, especially in cases where chronic meningitis may be suspected, is to exclude an alternative process such as an abscess, tumor, or parameningeal focus of infection. Although imaging studies usually exclude intramedullary brainstem processes, these studies often fail to yield abnormalities in extramedullary processes affecting cranial nerves. Therefore, the clinician is left to rely on the serologies as well as CSF abnormalities. See Table 4 for a complete list of serologic and CSF studies. Considering the broad differential diagnosis, a "shotgun" approach is not warranted and a directed approach guided by clinical history and examination is indicated.

CSF testing serves as an integral piece in the evaluation as the presence of a CSF pleocytosis further suggests the likelihood of an inflammatory, infectious, or neoplastic meningeal process. Unfortunately, simple CSF tests often fail to yield the diagnosis and more complex tests may be necessary. The type of CSF pleocytosis may help. Most of the meningeal processes discussed will cause a lymphocytic predominance. Although most often associated with parasitic disease, the presence of mild eosinophilia ( $>10 \%)$ may be helpful as eosinophilic meningitis has been associated with Coccidioides immitis, tuberculous meningitis, neurosyphilis, systemic lupus erythematosus (SLE), and leukemia/ lymphoma. ${ }^{4,51}$ A neutrophilic predominance has been associated with early tuberculous meningitis, fungal infections, and Behçet's disease. The finding of hypoglycorrhachia (decreased CSF glucose), suggests the 
Table 4 Possible Evaluation of Patients with Multiple Cranial Neuropathies

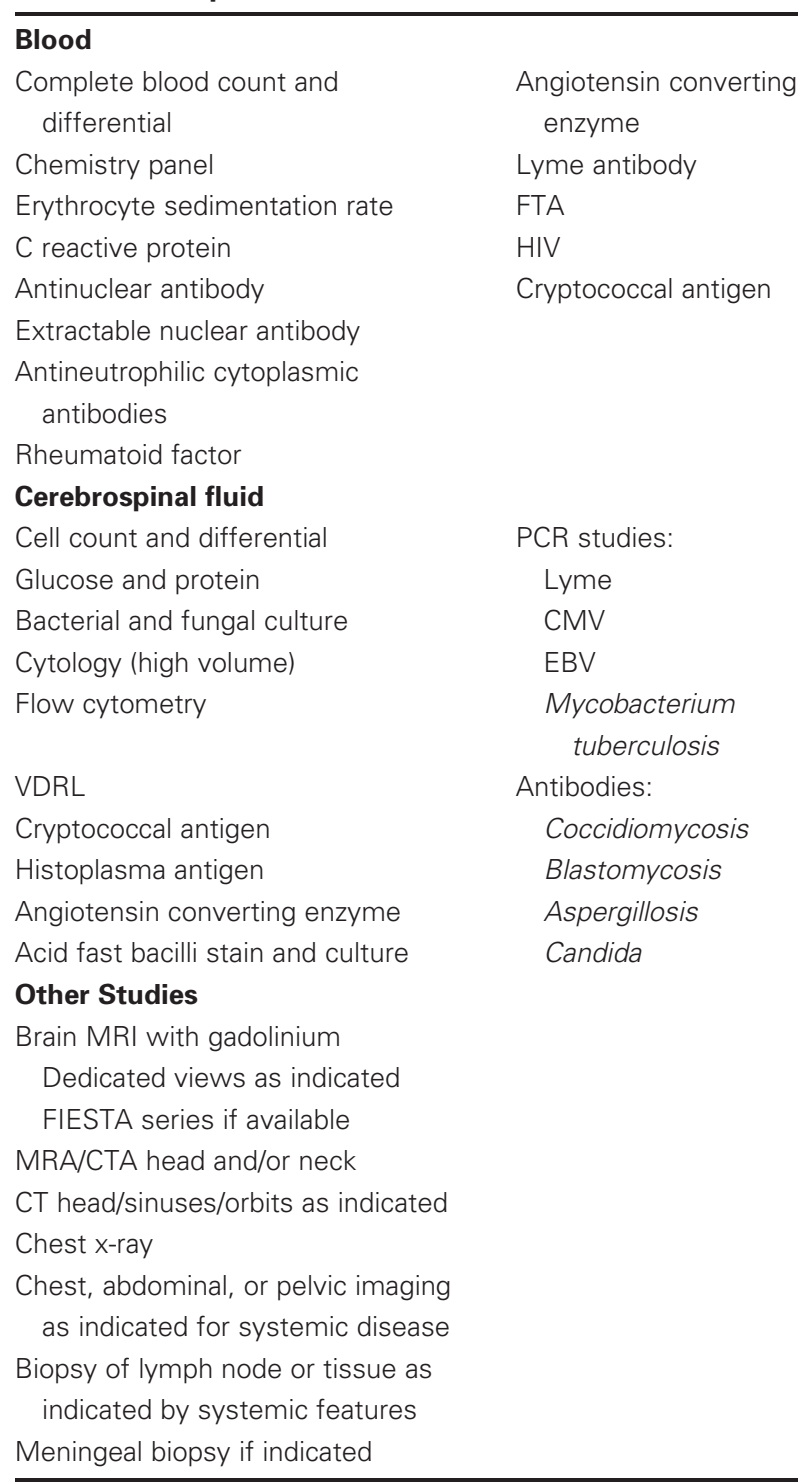

$\overline{\mathrm{CMV} \text {, cytomegalovirus; CT, computed tomography; CTA, computed }}$ tomography angiography; EBV, Epstein-Barr virus; FIESTA, fast imaging employing steady-state acquisition; FTA, fluorescent treponemal antibody; HIV, human immunodeficiency virus; MRA, magnetic resonance angiography; $\mathrm{MRI}$, magnetic resonance imaging: PCR, polymerase chain reaction; VDRL, venereal disease reference laboratory.

possibility of fungal, tuberculosis, or syphilitic meningitis. In addition, connective tissue diseases, sarcoidosis, and carcinomatous meningitis can also cause decreased CSF glucose. Multiple high-volume taps may be necessary for the diagnosis of carcinomatous, tuberculous, and fungal etiologies of chronic meningitis. Many experts agree that at least three high volume samples should be obtained if these processes are being considered. ${ }^{4}$ High volumes of CSF, at least 20 to $30 \mathrm{cc}$, should be sent to the laboratory, centrifuged, and the pellet examined microscopically, cultured and subjected to polymerase chain reaction $(\mathrm{PCR})$ analysis for whatever organisms are available. If the diagnosis remains obscure, repeated CSF sampling should be considered in this fashion. At least 10 to $20 \mathrm{cc}$ should be sent for cytologic evaluation. Flow cytometry should be performed when leukemic or lymphomatous meningitis is suspected as it may be more sensitive than conventional cytology. ${ }^{4}$ Analysis of cell lines may be very illuminating. If the cells are monoclonal, the process is likely neoplastic; if polyclonal, then infectious. C1-2 taps may be considered in lieu of highvolume lumbar taps. The point is to study the CSF at the base of the brain because that is where the pathology is. It is not in the CSF in the lumbar sac.

As already mentioned, routine imaging studies are often unremarkable. In cases where bony detail needs to be delineated, such as in craniomaxillofacial trauma or in cases of neoplasms with erosion or tumor extension into cranial foramina, computed tomography (CT) scans may be necessary. This is particularly true in skull base lesions. Furthermore, skull base lesions often also involve the extracranial and intracranial soft tissues; as such, MRI of the neck is superior to CT in evaluating the soft tissue structures. In cases where a clinical suspicion exists for a suprasellar mass or cerebellopontine angle mass, MRI with dedicated pituitary or internal auditory canal cuts should be obtained. In patients presenting with ophthalmoplegia, CT scans or contrast-enhanced MRI scans with orbital views may also be necessary. If vascular pathology is considered, then CT angiography (CTA) or MRA of the head and/or neck should be obtained. This is particularly true when a cavernous sinus localization is considered where vascular imaging would best demonstrate potential evidence for cavernous thrombosis, aneurysms, or carotid-cavernous fistulas. Due to the small diameter and complex anatomic course of the cranial nerves, many are difficult to image. In addition to standard MRI, newer imaging sequences may increase the sensitivity of visualizing the cranial nerves. The three-dimensional fast imaging employing steady-state acquisition (3D-FIESTA) method is a fast 3D steadystate imaging sequence that provides higher spatial resolution of these structures and increases the ability to visualize the cranial nerves, and detect abnormalities in their course. ${ }^{52-54}$ If the evaluation reveals evidence for tumor, a search for the primary source of neoplasia may be necessary including chest $\mathrm{x}$-ray, or chest, abdominal, and pelvic $\mathrm{CT}$ scans, or a positron emission tomography (PET) scan. Even in nonneoplastic processes, these studies may reveal evidence for a more widespread systemic process, enlarged lymph nodes or other abnormalities, or a lesion that can be biopsied for diagnosis.

If all the aforementioned investigations are futile, a meningeal biopsy must be considered if a chronic meningitis, neoplastic process or CNS vasculitis is suspected. ${ }^{55-59}$ Even a leptomeningeal biopsy may not yield a definitive diagnosis. In a study examining the role of meningeal biopsy in 25 patients with meningitis, the 
biopsy was diagnostic in only five patients: Mycobacterium tuberculosis was found in one, neoplasia in three, and granulomatous angiitis in one patient. ${ }^{59}$ The diagnostic yield of meningeal biopsy can be increased by targeting regions that enhance with contrast on MRI. ${ }^{58}$ In another study of patients with chronic meningitis, a definite diagnosis by meningeal biopsy was possible in only $39 \%$. The yield increased to $80 \%$ if the biopsy was obtained from an enhancing area noted on the MRI. ${ }^{58}$ The yield without MRI enhancement was only $10 \%$. A repeat biopsy was obtained in four patients and revealed adenocarcinoma, sarcoidosis, demyelinating disease, and chronic inflammation in one patient each. ${ }^{58}$ The yield of the biopsy is slightly higher when obtained from the posterior fossa than from the cerebral cortex, and if possible, should include both the meninges and underlying brain. Common diagnoses made from biopsies include neurosarcoidosis, hypertrophic pachymeningitis, leptomeningeal metastasis, vasculitis, and infections. ${ }^{4}$ Depending on the clinical suspicion, a portion of the biopsy should be cultured or assayed by PCR for fungi, Mycobacterium tuberculosis, and uncommon bacteria. ${ }^{4} \mathrm{At}$ times, granulomatous changes in meningeal biopsy specimens may lead to diagnostic confusion with neurosarcoidosis in culture-negative fungal meningitis. ${ }^{60}$ Rarely, repeat biopsies may be indicated for clinical deterioration.

\section{MANAGEMENT}

The management of multiple cranial neuropathy palsies relies on accurate diagnosis with specific therapy aimed at the underlying cause. For example, if an infectious cause is found, appropriate antimicrobial therapy is indicated. Detailed management for the multitude of specific etiologies is beyond the scope of this discussion. Although therapeutic decisions may seem somewhat straightforward when an etiology is found, a lack of a definable etiology presents a management challenge. When an extensive workup fails to yield a diagnosis, the clinician is often left feeling hopeless, without a directed plan. This is not an uncommon dilemma. Despite an extensive evaluation, 15 to $30 \%$ of patients with chronic meningitis do not have a clear etiology. ${ }^{4}$ Due to the vast array of causes, the complex nature of the evaluation, and the lack of evidence-based guidelines, it is very difficult to offer a straightforward algorithm for the management of these patients. One is often faced with the question of whether or not to start empiric therapy. In patients presenting with rapid progression of signs and symptoms, empiric therapy seems reasonable. In patients with severe meningitis with risk factors for tuberculosis, empiric antituberculous medications are usually recommended even before final laboratory results or cultures are obtained because serious neurologic sequelae may develop if the patient is not treated promptly. ${ }^{4}$ Likewise, if the patient has risk factors for a fungal meningitis, treatment with fluconazole should be considered. Often a decision must be made whether or not to start empiric steroids. From an evidence-based view, this answer remains elusive. Considering that neuroimaging will exclude many neoplastic processes (particularly skull-based tumors), vascular disorders and bone diseases, the clinician is often left with considerations of an infectious versus an inflammatory process. When the CSF reveals a pleocytosis indicative of meningeal inflammation and every attempt to exclude infectious causes has been made, then inflammatory etiologies, which often respond to corticosteroids, become high on the differential. Therefore, despite the lack of evidence-based answers, empiric corticosteroids seem a logical management option. In one series of 49 patients with chronic idiopathic meningitis in whom no etiology was found, $52 \%$ responded symptomatically to empiric corticosteroids. Despite symptomatic improvement, no effect on ultimate outcome was demonstrated, as $85 \%$ of those without a clear diagnosis had a benign long-term course. ${ }^{61}$ Considering the lack of literature to support this decision, specific recommendations regarding dosing cannot be made. If there are severe signs, it may be reasonable to start with high-dose intravenous (IV) Solu-Medrol ${ }^{\circledR}$ (Pfizer Pharmaceuticals, New York, NY) $1000 \mathrm{mg}$ daily for 3 to 5 days followed by highdose oral prednisone with further dosing taper-based on clinical response. In cases of vasculitis or other rheumatologic disease, alternative immunosuppressants such as cyclophosphamide can be considered; however, one should be reluctant to initiate such an aggressive therapy in the absence of a clear diagnosis. In these cases, a meningeal biopsy prior to initiation of therapy should be considered.

\section{CONCLUSION}

The patient presenting with multiple cranial nerve palsies remains a formidable challenge to any physician evaluating this clinical presentation. The differential diagnosis is extensive and the workup can be daunting with expensive and invasive tests. The stakes can be very high with progressive accumulation of significant neurologic disability. The evaluation requires a systematic yet targeted approach guided by clinical history and examination. Although many of the causes have specific therapies, the evaluation often leads to a dead end requiring individual clinical discretion to decide on the best possible empiric therapy.

\section{REFERENCES}

1. Campbell WW. Brainstem and multiple cranial nerve syndromes. In Campbell WW, ed. Dejong's The Neurologic Examination. 6th ed. Philadelphia: Lippincott Williams and Wilkins; 2005:277-296 
2. Keane JR. Multiple cranial nerve palsies. Arch Neurol 2005; 62:1714-1717

3. Beal MF. Multiple cranial nerve palsies: a diagnostic challenge. N Engl J Med 1990;322:461-463

4. Davis L. Subacute and chronic meningitis. Continuum Lifelong Learning in Neurol 2006;12(2):27-57

5. Cohen BA. Chronic meningitis. Curr Neurol Neurosci Rep 2005;5:429-439

6. Reik L Jr, Burgdorfer W, Donaldson JO. Neurologic abnormalities in Lyme disease without erythema chronicum migrans. Am J Med 1986;81:73-78

7. Anderson NE, Willoughby EW. Chronic meningitis without predisposing illness: a review of 83 cases. QJ Med 1987; 63:283-295

8. Thwaites GE, Tran TH. Tuberculous meningitis: many questions, too few answers. Lancet Neurol 2005;4:160-170

9. Leonard JM, Dez Prez RM. Tuberculous meningitis. Infect Dis Clin North Am 1990;4:769-787

10. Davis LE, Rastogi KR, Lambert LC, Skipper BJ. Tuberculous meningitis in the southwest United States: a community-based study. Neurology 1993;43:1775-1778

11. Juncos JL, Beal MF. Idiopathic cranial polyneuropathy: a fifteen year experience. Brain 1987;110:197-211

12. Girkin CA, Perry JD, Miller RN, Reich SG. Pachymeningitis with multiple cranial neuropathies and unilateral optic neuropathy secondary to Pseudomonas aeruginosa: Case report and review. J Neuroophthalmol 1998;18:196-200

13. Marra CM. Human immunodeficiency virus. In: Scheld WM, Whitley RJ, Marra CM, eds. Infections of the Central Nervous System. 3rd ed. Philadelphia: Lippincott Williams \&Wilkins; 2004:271-286

14. Calado S, Canas N, Viano-Baptista M, Ribeiro C, Mansinho K. Multiple cranial neuropathy and HIV-2. J Neurol Neurosurg Psychiatry 2004;75:660-661

15. Mayo DR, Booss J. Varicella zoster-associated neurologic disease without skin lesions. Arch Neurol 1989;46:313-315

16. Landgren M, Kyllerman M, Bergstrom T, Dotevall L, Ljungstrom L, Ricksten A. Diagnosis of Epstein-Barr virusinduced central nervous system infections by DNA amplification from cerebrospinal fluid. Ann Neurol 1994;35:631-635

17. Masdeu JC, Small CB, Weiss L, Elkin CM, Llena J, MesaTejada R. Multifocal cytomegalovirus encephalitis in AIDS. Ann Neurol 1988;23:97-99

18. Ceriotto M, Zala C, Brandariz S, et al. Trypanosoma cruzi encephalitis (Chagas' disease) in a patient with HIV infection. Abstract presented at: XI International Conference on AIDS; 1996; July 7-12; Vancouver (abstract no. Tu.B.2211)

19. Gullapalli D, Phillips LH II. Neurologic manifestations of sarcoidosis. Neurol Clin 2002;20:59-83

20. Hoitsma E, Farber CG, Drent M, Sharma OP. Neurosarcoidosis: a clinical dilemma. Lancet Neurol 2004;3:397407

21. Stern BJ. Neurological complications of sarcoidosis. Curr Opin Neurol 2004;17:311-316

22. Scott TF. Neurosarcoidosis: progress and clinical aspects. Neurology 1993;43:8-12

23. Akman-Demir G, Serdaroglu P, Tasci B. Clinical patterns of neurological involvement in Behcet's disease: evaluation of 200 patients. Brain 1999;122:2171-2181

24. Siva A, Altintas A, Saip S. Behcet's syndrome and the nervous system. Curr Opin Neurol 2004;17:347-357

25. Nishino H, Rubino FA, DeRemee RA, Swanson JW, Parisi JE. Neurological involvement in Wegener's granulomatosis: an analysis of 324 consecutive patients at the Mayo Clinic. Ann Neurol 1993;33:4-9

26. Nadeau SE. Neurologic manifestations of systemic vasculitis. Neurol Clin 2002;20:123-150

27. Case Records of the Massachusetts General Hospital (Case 8-1995). N Eng1 J Med 1995;332:730-737

28. Younger DS. Neurovasculitis. Continuum Lifelong Learning in Neurol 2005;11(1):11-42

29. Katzenstein AL, Carrington CB, Liebow AA. Lymphomatoid granulomatosis: a clinicopathologic study of 152 cases. Cancer 1979;43:360-373

30. Mori K, Iijima M, Koike H, et al. The wide spectrum of clinical manifestations in Sjögren's syndrome-associated neuropathy. Brain 2005;128(pt 11):2518-2534

31. Lewis S. Neurologic complications of Sjögren's syndrome and rheumatoid arthritis. Continuum Lifelong Learning in Neurol 2008;14:120-144

32. Nadeau SE. Neurologic manifestations of connective tissue disease. Neurol Clin 2002;20:151-178

33. Touze E, Blanche P, Zuber M. A 35 year history of recurrent multiple cranial neuropathy due to primary Sjögren's syndrome. J Neurol 1999;246:968-969

34. Masson C, Boukriche Y, Colombani JM. Inflammatory hypertrophic cranial pachymeningitis. Presse Med 2001;30: 411-416

35. Hamilton SR, Smith CH, Lessell S. Idiopathic hypertrophic cranial pachymeningitis. J Clin Neuroophthalmol 1993;13: 127-134

36. Kyle RA, Dyck PJ. Amyloidosis and neuropathy. In: Dyck PJ, Thomas PK, eds. Peripheral Neuropathy. 3rd ed. Philadelphia: WB Saunders; 1993:1294-1309

37. Van Gerpen JA, Goudreau JL, Dodick DW, Gertz MA. Amyloidosis presenting with intractable epistaxis and multiple cranial neuropathies. Neurology 2000;55:1755-1756

38. Demopoulos A. Leptomeningeal metastasis. Curr Neurol Neurosci Rep 2004;4:196-204

39. Kim L, Glantz MJ. Neoplastic meningitis. Curr Treat Options Oncol 2001;2:517-527

40. Chamberlain MC. Neoplastic meningitis. J Clin Oncol 2005; 23:3605-3613

41. Lachance DH, O'Neill BP, MacDonald DR, et al. Primary leptomeningeal lymphoma: report of three cases, diagnosis with immunocytochemical analysis, and review of the literature. Neurology 1991;41:95-100

42. Davila G, Duyckaerts C, Lazareth JP, Poisson M, Delattre JY. Diffuse primary leptomeningeal gliomatosis. J Neurooncol 1993;15:45-49

43. Mokri B, Silbert PL, Schievink WI, et al. Cranial nerve palsy in spontaneous dissection of the extracranial internal carotid artery. Neurology 1996;46:356-359

44. Eshbaugh CG, Siatkowski RM, Smith JL, et al. Simultaneous, multiple cranial neuropathies in diabetes mellitus. J Neuroophthalmol 1995;15:219-224

45. Asher SW. Multiple cranial neuropathies, trigeminal neuralgia, and vascular headaches in sickle cell disease, a possible common mechanism. Neurology 1980;30:210 211

46. Shapiro F. Osteopetrosis. Current clinical considerations. Clin Orthop Relat Res 1993;(294):34-44

47. Poncelet A. The neurologic complications of Paget's disease. J Bone Miner Res 1999;14:88-89

48. Manni JJ, Scaf JJ, Huygen PLM, Cruysberg JRM, Verhagen WIM. Hyperostosis cranialis interna: a new hereditary 
syndrome with cranial-nerve entrapment. N Engl J Med 1990;322:450-454

49. Bone I, Hadley DM. Syndromes of the orbital fissure, cavernous sinus, cerebellopontine angle, and skull base. J Neurol Neurosurg Psychiatry 2005;76(Suppl III):iii29-iii38

50. Polo A, Manganotti P, Zanette G, De Grandis D. Polyneuritis cranialis: clinical and electrophysiological findings. J Neurol Neurosurg Psychiatry 1992;55:398-400

51. Fishman RA. Composition of the cerebrospinal fluid. In: Fishman RA, ed. Cerebrospinal Fluid in Diseases of the Nervous System. 2nd ed. Philadelphia: WB Saunders Company; 1992:194

52. Mikami T, Minamida Y, Yamaki T, Koyanagi I, Nonaka T, Houkin K. Cranial nerve assessment in posterior fossa tumors with fast imaging employing steady-state acquisition (FIESTA). Neurosurg Rev 2005;28:261-266

53. Okumura Y, Suzuki M, Takemura A, et al. Visualization of the lower cranial nerves by 3D-FIESTA. Nippon Hoshasen Gijutsu Gakkai Zasshi 2005;61:291-297

54. Cheng YS, Zhou Z, Peng WJ, Tang F. Three-dimensionalfast imaging employing steady-state acquisition and T2weighted fast spin-echo magnetic resonance sequences on visualization of cranial nerves III-XII. Chin Med J (Engl) 2008;121:276-279

55. Volcy M, Toro ME, Uribe CS, Toro G. Primary angiitis of the central nervous system: report of five biopsy-confirmed cases from Colombia. J Neurol Sci 2004;227:85-89

56. Sylaja PN, Cherian PJ, Das CK, et al. Idiopathic hypertrophic cranial pachymeningitis. Neurol India 2002;50:53-59

57. Jinnah HA, Dixon A, Brat DJ, Hellmann DB. Chronic meningitis with cranial neuropathies in Wegener's granulomatosis. Case report and review of the literature. Arthritis Rheum 1997;40:573-577

58. Cheng TM, O'Neill BP, Scheithauer BW, et al. Chronic meningitis; the role of meningeal or cortical biopsy. Neurosurgery 1994;34:590-595

59. Anderson NE, Willoughby EW, Synek BJ. Leptomeningeal and brain biopsy. Aust N Z J Med 1995;25:703-706

60. Thompson GR III, Lavalle CE III, Everett D. Unusual manifestations of histoplasmosis. Diagn Microbiol Infect Dis 2004;50:33-41

61. Smith JE, Aksamit AJ. Outcome of chronic idiopathic meningitis. Mayo Clin Proc 1994;69:548-556 